\title{
Interaction design of automatic faucet for standard hand-wash
}

\author{
Hurriyatul Fitriyah*, Edita Rosana Widasari, Eko Setiawan, and Brian Angga Kusuma \\ Dept. of Computer Engineering, Faculty of Computer Science, University of Brawijaya, Indonesia
}

\begin{abstract}
In hand-washing practice, people tend to forget to use soap and scrub for 20 seconds as stated in the standard. This paper attempts to develop an automatic faucet that is user-friendly and easy to plug in regular water pipe for standard hand-washing routine. An Interaction Design Process that aims to maximize product usability was applied during the development. The faucet utilized IR proximity sensor to detect presence of hands that would automatically commence the hand-washing process. First, it exited water and soap simultaneously to force users to use soap. The scrubbing duration was marked by buzzer's sound and LED's light. The validation test showed health practitioner agreed the faucet facilitates a standard handwashing. The user test in 30 participants showed users used soap and scrubbed in the exact duration. The usability questionnaire filled by participants showed they were strongly agreed for its usefulness and agreed for its satisfaction and easiness.
\end{abstract}

\section{Introduction}

Hand hygiene is a major requirement for human health. Many infectious diseases can be emerged if proper hand hygiene procedures are not implemented [1, 2]. The infectious diseases, which are caused by hand nonhygiene, are viruses (hepatitis A, polio, diarrhoea), bacteria (typhoid, fever, dysentery), and parasites (amoeba dysentery, enterobijaza) [3]. Furthermore, the impact of hand non-hygiene can increase pandemic public health such as severe acute respiratory syndrome and avian influenza in the community [4]. Many studies have also been reported an association between improvements in hand hygiene and reductions in rates of infectious illness. The Medline database was searched from January 1980 to June 2001 shows the relation between hygiene practices and relative reduction in risk of illness is greater than $20 \%[5,6]$.

Hand washing is the simplest, important and costeffective way to improve hand hygiene in health care and support the prevention of infectious disease. The WHO standard obligates people for hand washing with nonantibacterial soap and water. The duration ranged on average as short as 20 seconds to 30 seconds, including rub the backs of hands, wrists, between fingers and under fingernails $[1,7]$. Hand washing with soap and water is more effective for the removal of bacteria than washing with water alone to $23 \%$ [8].

Studies show that the compliance of hand-washing procedures by healthcare workers, householder and officer are only about $40 \%$, and then barely reaches $50 \%$ even after various educational trainings or interventions [9]. A study also showed that the average duration of scrubbing was less than 15 seconds in the community [10].
Interaction design process aims to maximize usability of a product. It focuses not only on functionality, but userfriendliness of a product as well. It consists of 5 steps which are: (1) What is wanted, (2) Analysis, (3) Design, (4) Prototype, (6) Implementation and Deployment [11]. Analysis-Design-Prototype is iterative process, where the product kept redesigned until the product requirements are met. The Interaction design process had been applied widely not only to design a software application but computer hardware as well $[12,13]$.

\section{Related Work}

In market, automatic faucets that do not necessitate users to turn the faucet's lever to exit water are common. It uses proximity sensor that mostly based on infrared (IR) light to detect presence of hands. Several automatic faucets to exit soap also have used the similar usage of proximity sensor in detecting hands.

In term of designing automatic system for handwashing standard procedure, [14] has developed a machine that accommodate wetting, lathering, scrubbing, rinsing and drying in automatic mode. The machine consisted of a sink and 3 faucets of water, soap, air blower that was controlled electronically. The hand-wash process used timer to exit soap, then water and then air blow. The design also included mechanical component of water piping, pump and housing. Other patented design entitled Automatic Hand Washing and Drying Machine were also consisted of water-soap-dryer [15]. The machine design included electronic components and mechanical piping and pump.

Both designs were in a form of integrated automatic basin. Both machines were required to be assembled as a

\footnotetext{
* Corresponding author: hfitriyah@ub.ac.id
} 
complete basin system. In market, such automatic basins were available as well.

Instead of developing a complete basin for standard hand-wash, this paper aim to develop an automatic handwash faucet, which can be used simply by replacing the standard faucet with the developed one. It was designed for simpler solution of performing hand-wash standard procedure instead of buying the whole housing of handwash machine.

\section{Methodology}

Development of the automatic faucet used Interaction Design process to carefully consider the usability of the product that meet the standard hand-wash procedure as well.

\subsection{What is Wanted}

The very first step in interaction design is "What is Wanted". The developer must first enlist the exact definition or information of the product. The definition consists of product's functionality, environment, users or any expectation that should be met by the product.

- Definition of Product: (1) The faucet is equipped with soap, (2) It is a free-hand operation, (3) It must obey the hand-washing standard procedure.

- Definition of Environment: A wet environment, hence electronically components must be protected.

- Definition of Users: General users

There are two important notes from the hand-washing procedure that must be obeyed in designing the automatic faucet, which are:

\subsubsection{The automatic faucet force users to use soap}

Washing hands with soap is compulsory. Although it is not necessarily an antibacterial soap, a regular soap is required since the surfactants in soap could lift soil and microbes from skin. Using antibacterial soap might even lead to development of bacteria that are resistant to the product's antimicrobial agents [16]. Due to low percentage of users that use soap in their hand-washing routine, hence the automatic faucet must be designed to force the users to use soap with no exception.

\subsubsection{The automatic faucet force must guide users for exact 20 seconds duration of scrubbing}

The 20 seconds duration of scrubbing is important. CDC mentioned that the duration was necessarily required to kill bacteria in hands. Hence, the automatic faucet must be designed to necessitate the users to scrub in the exact duration.

\subsection{Analysis}

The "Analysis" step is a formulation of the "What is Wanted". It comprises structured Scenario and Task Analysis. Scenario is a story that contains visual description of how users would interact with the developed product. Task Analysis is a structured task decomposition of how users would perform the task using the developed product in a chronological order.

\subsubsection{Scenario}

The story of how a user would wash their hands using the developed automatic faucet is descripted below:

"Adam was about to having a dinner. His hand was dirty since he has spent his afternoon for gardening in his backyard. He then walk into his fully automatic handwashing faucet and put his hand beneath. The presence of his hand automatically makes water ran from the end of the faucet along with soap. Realising there is soap in his hand, he has no choice than to wash his hands using the soap, the thing he usually forgot to use. He then lathered his hand and scrubs it few seconds until the faucet released water automatically for once more. He rinsed the soap from his hand and dried his hand with towel"

\subsubsection{Task analysis}

From the description in the "Scenario", the task of washing hands using the automatic faucet was structured in hierarchy. The hierarchy is named Hierarchical Task Analysis (HTA) that contains task decomposition in chronological orders. The HTA of the developed automatic faucet is composed below:

0 . Washing hand with soap using the automatic faucet

1. Put hands under the faucet's end to exit water and soap simultaneously

2. Lather and scrub the hands with soap until the water run once more

3. Rinse the hands thoroughly

Plan 0: do $1-2-3$ in that order

\subsection{Design}

The "Design" process consists of designing the product's System and Applying Design Principle. In this paper, the System includes Structure, Hardware Design and Housing Design. The Design principle was applied as a checklist of design characteristic that is advised to be fulfilled to maximize product's usability.

\subsubsection{Structure}

To perform the task as mentioned in the HTA above, the system of automatic faucet was designed to run in chronological order as well. The product's structures are: (1) Exit water \& soap, (2) Stop water \& soap, (3) Wait 20 seconds for scrubbing, (4) Exit water for rinsing, (5) Stop water.

\subsubsection{Hardware design}

The product's hardware was designed according to the 5 structures of the system. It was controlled electronically. Its operation was initiated by the presence of hands. 
Proximity sensors are common sensor used for the role. An infrared-based proximity sensor was used in this paper since it would strictly detect the motion of human's hands. Once the hands are detected, the sensor sent signal to a microcontroller to start the opening of valves by activating a motor. At first, the valve would open two inlets, which are water's inlet and soap's inlet. This opening would make the faucet exited water and soap simultaneously. The water and soap were programmed to be exited for 3 seconds, a sufficient duration to wet the hands and lather the soap thoroughly.

The product then proceeds for scrubbing phase. The microcontroller instructed the motor to close the inlets of water and soap to stop the water and soap from running down the faucet. The closing is programmed for 20 seconds, the exact duration necessarily required for scrubbing according to the hand-washing procedure. A buzzer and LED lamp was activated during this process to make users intuitively start the scrubbing. It programmed to beep and flashed in accelerated intervals giving users guidance when the duration was about to end.

The product then proceeds for rinsing phase. The microcontroller instructed the motor to open the water's inlet only. The opening was programmed for 10 seconds, a sufficient duration for cleaning the soap form hands thoroughly. The Flow Diagram of the automatic faucet is shown in Figure 1.

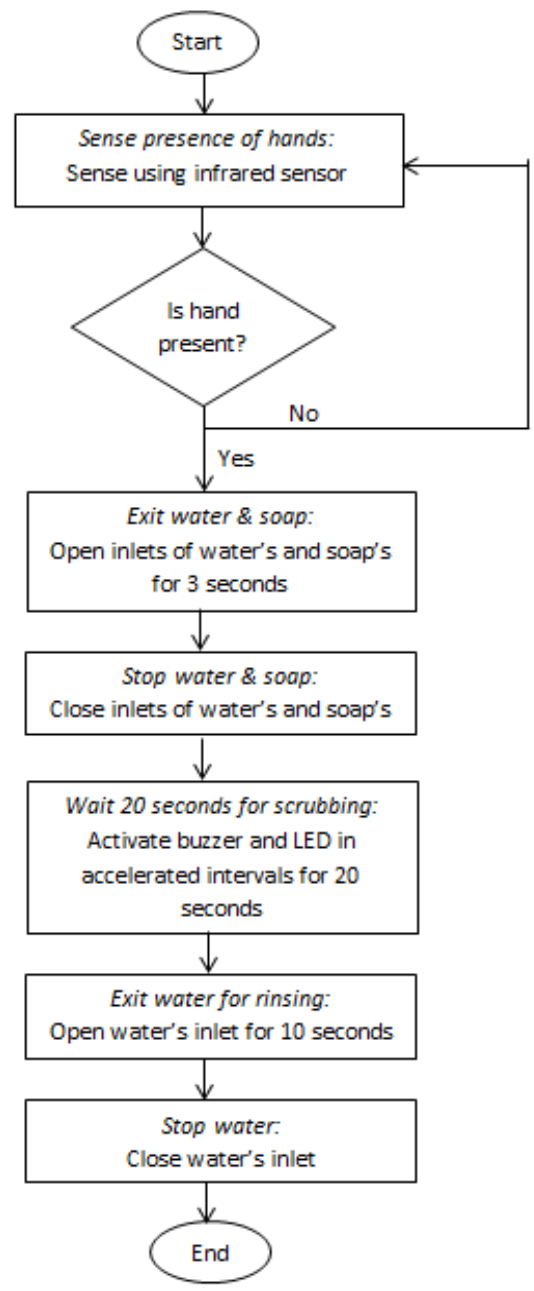

Fig 1. The flow diagram of the automatic faucet
From the Flow Diagram in Figure 1, the product's hardware consisted of IR proximity sensor as input, a microcontroller as controller, buzzer and motor as outputs. The block diagram is shown in Figure 2.

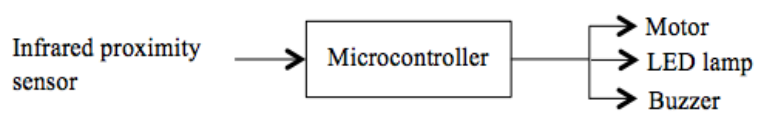

Fig 2. Block diagram of the automatic faucet's hardware

\subsubsection{Housing design}

From the Hardware Design, the automatic faucet required 6 components of Housing, which are: (1) Soap container, (2), Water inlet, (3) Box for Microprocessor, (4) Box for motor, (5) Sliding lid to cover water and soap's inlet, (6) Water Outlet. Each component followed below requirements:

- Soap container: Tube shape. Soap outlet at the bottom. Refillable

- Water inlet: Standard connection size and type with water pipe

- Box for microprocessor: Waterproof to protect the electronics component

- Box for motor: Waterproof to protect the electronics component

- Sliding lid: Leak-proof material to cover 2 inlets, water and soap. The lid was full-opened to exit water and soap and half-opened to exit water only

- Water outlet: Standard pipe that run water or soap from top to bottom of the tip.

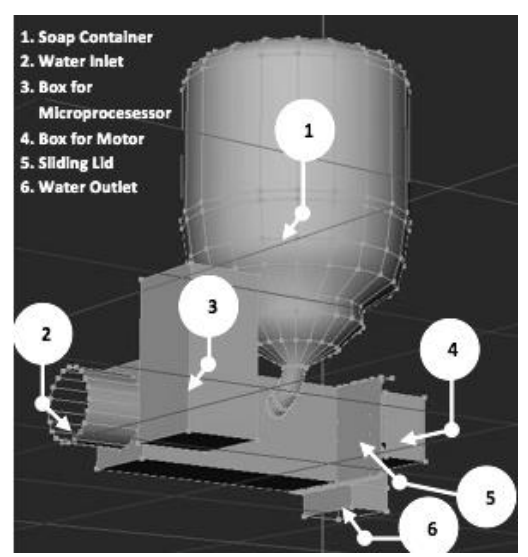

Fig 3. The housing design of automatic faucet

The components were set in a configuration as shown in Figure 3. The motor was placed close to the sliding lid, where it would open and close the passage of water and soap. The microcontroller was hidden in the back.

\subsubsection{Applying design principle}

The Design Principles consist of Learnability, Flexibility and Robustness. Learnability, which consists of Predictability, Synthesizability, Familiarity, Generality, Consistency, is about designing an easy and intuitive 
operation of the product. Flexibility, which consists of Dialogue Initiative, Task Migratability, Multi-Threading, Subtitutivity and Customizability, is about designing a friendly and satisfied experience when using the product. Robustness, which consists of Observability, Responsiveness, Task Conformance, Recoverability, is about designing a useful product that also able to handle human's error. These principles are applied in designing the interaction of developed automatic faucet.

a) Learnability

- Predictability: The developed faucet did not equipped with lever similar to other automatic faucet; hence users intuitively put their hands under the faucet's tip. An upturned hands icon was also placed on the tip for guiding users to put their hands beneath it to activate the automatic faucet. Once the hand had been detected, the faucet exited water and soap simultaneously. Seeing soap in their hands, users could easily predict the next action, which was scrubbing their hands with it.

- Synthesizability: The water and soap was designed to come out once the hands were detected; hence users able to correlate the faucet would commence the handwashing process by placing their hands beneath the faucet's tip.

- Familiarity: The developed faucet had icon of upturned hands on its tip as guidance for users to intuitively put their hands beneath it. This simple hands symbol in upturned position is easily understandable by users. The hands icons were also accompanied by water-drop and bubble icon for users to expect the water and soap to come out of the tip. The symbol is shown in Figure 4.

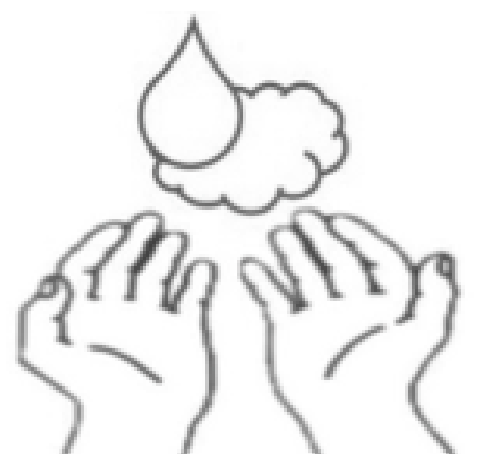

Fig 4. The icon of water drop and bubble

- Generality: The developed faucet shared general shape with common faucets. It had tip where users expect waters to come out from. It used standard water-pipe connector. The soap container shape was also a common tube where the refill access was put upside down.

- Consistency: Every hand-washing process in the developed faucet worked consistently based on timer. No manual operation was needed in the process.

b) Flexibility

- Dialogue Initiative: The developed faucet was designed for fully automatic and standardized operation; hence the faucet led the interaction. Users only initiate the interaction by placing their hands beneath the faucet's tip. This dialogue arrangement was designed for operation simplicity.

- Task Migratability: The developed faucet was designed for fully automatic operation; hence users do not have to perform a task of turning a lever to run the water and pressing the soap pump to run the soap such in common faucet's operation. Users also does not have to count for exact 20 second or singing "Happy Birthday" song as suggested in scrubbing phase, since the faucet provided 20 -seconds-waiting for the process accompanied with buzzer's beep and LED's flash light.

- Multi-threading: The developed faucet was designed for simple hand-washing in standard procedure, hence no multi-threading is needed.

- Subtitutivity: The developed faucet works on common behaviour similar to other automatic faucets. The input is simply from the presence of hands only.

- Customizability: The developed faucet was designed for standardized hand washing. It is also utilized for shared-using. A customization based on users' preference was not necessary.

c) Robustness

- Observability: During the scrubbing process, users may observe the timing by listening to buzzer's beep that was programmed to sounds in accelerated fashion. An LED lamp was also programmed to flash synchronously to the buzzer's beep. Both buzzer's beep and LED's flash was applied to intuitively guide the users to scrub their hands in exactly 20 seconds. This feedback was also a guarantee to users that the faucet was working a progress and not just silently broken in the 20 seconds duration.

- Responsiveness: For fast response, the developed faucet's program used timer. Developer determined the 3 seconds duration of wetting-leathering and 10 seconds duration of rinsing based on trials.

- Task Conformance: The developed faucets worked as intended, which to assist users to perform a hand washing in standardize and suggested procedure.

- Recoverability: Although a reset button was a good tool for a user to re-do the operation when previous user abandoned the faucet in the middle of process, it was not provided in the faucet for two reasons. First reason was that the process only last for less than 1 minute. Second reason was that adding a reset button would burden the responsiveness due to hardware and software complexity.

d) Prototype

A prototype of the faucet was developed using an Adjustable IR Reflection Sensor as input to detect presence of hands; Microcontroller AT8353 as controller; DC motor, LED lamp and 6V round electrical buzzer as outputs. These components were connected as shown in Figure 5. Once the hands were detected by IR sensor, it sent digital signal 1 to the microcontroller. The microcontroller then started the program to control motor, LED lamp and buzzer according to the designed Block Diagram in Figure 1. 


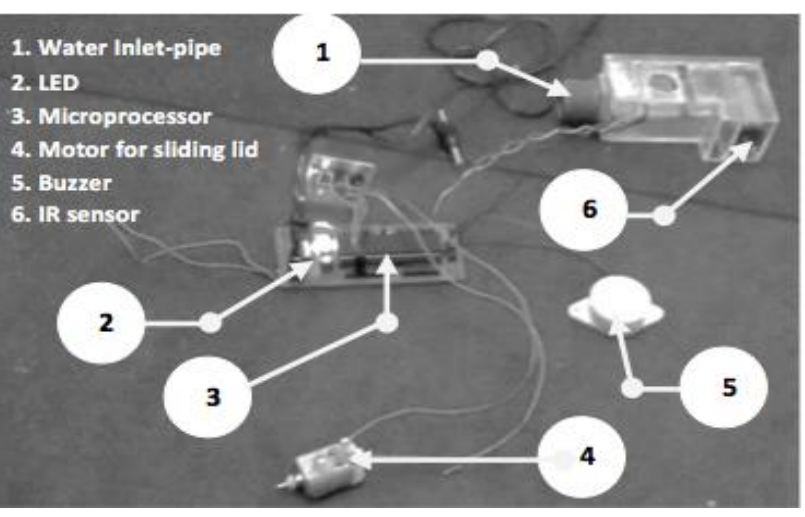

Fig 5. Prototype of the automatic faucet

The electrical circuit were then integrated with the designed Housing in Figure 3 to result an automatic faucet as shown in Figure 6. The case was made using acrylic as it is cheap and easy material to cut and glued. The water's inlet used a standard connector of PVC pipe. The soap container used a plastic bottle. The sliding lid was also made using acrylic, which was sealed by rubber for leakproof guard.

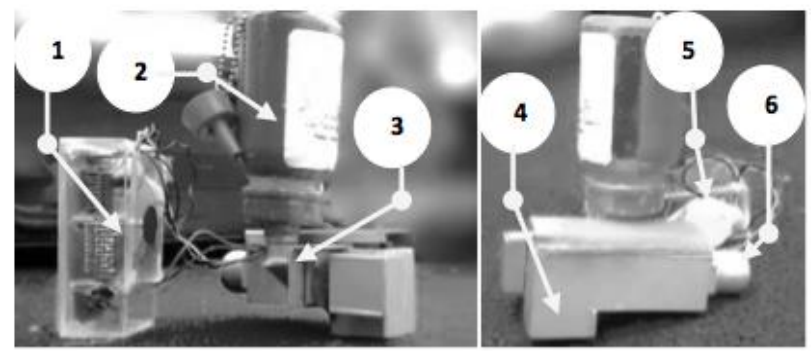

1. Microprocessor Box; 2. Soap Container; 3. Sliding Lid

4. Outlet pipe; 5. Buzzer; 6. Water-inlet pipe

Fig 6. The integrated case of an automatic faucet

\section{e) Implementation and development}

The Implementation and Deployment step includes designing Product Documentation and User Support. In this paper, both were bundled in a form of User's Manual Book. It consisted of:

- Installation Guide: Instruction on assembling the water inlet to water pipe

- Device Layout: Diagrammatic layout of device's part

- Operation Manual: Step by step instruction accompanied with pictures on how to perform handwash using the developed automatic faucet.

- Maintenance: Advice on caring the water inlet and soap outlet

- Troubleshooting: What-to-do instruction on handling jammed motor and microcontroller

\section{Testing Results and Discussion}

Three tests are performed on the developed automatic faucet, which are Verification, Validation and UserParticipation Test.

\subsection{Verification on faucet functionality}

The verification on faucet functionality was performed to find the performance of: (1) Distance of adjustable IR reflection sensor. The result in Table 1 shows the minimum distance that can be detected by the sensor was $3 \mathrm{~cm}$ and the maximum distance was $80 \mathrm{~cm}$. This distance was reasonable distance to detect presence of human hands beneath the automatic faucet's tip. (2) DC Motor connectivity. The result in Table 2 shows the DC motor could drive the sliding lid to fully open, half open and fully closed the inlets of water and soap. (3) Microcontroller to buzzer and LED lamp connectivity. The Microcontroller was able to function the Buzzer and LED in accelerated fashion within 20 seconds.

Table 1. Distance of adjustable IR refection sensor.

\begin{tabular}{|c|c|}
\hline Distance (cm) & LED Lamp (as Indicator) \\
\hline 3 & ON \\
\hline$>80$ & OFF \\
\hline
\end{tabular}

Table 2. DC motor connectivity.

\begin{tabular}{|c|c|c|}
\hline Variable & Movement & Result \\
\hline Full Open & $\begin{array}{c}\text { DC motor drived the } \\
\text { sliding lid in full rotation } \\
\text { counter-clockwise }\end{array}$ & $\begin{array}{c}\text { The automatic } \\
\text { faucet exited water } \\
\text { and soap } \\
\text { simultaneously }\end{array}$ \\
\hline $\begin{array}{c}\text { Half } \\
\text { Open }\end{array}$ & $\begin{array}{c}\text { DC motor drived the } \\
\text { sliding lid in half rotation } \\
\text { counter-clockwise }\end{array}$ & $\begin{array}{c}\text { The automatic } \\
\text { faucet exited water } \\
\text { only }\end{array}$ \\
\hline $\begin{array}{c}\text { Full } \\
\text { Close }\end{array}$ & $\begin{array}{c}\text { DC motor drived the } \\
\text { sliding lid in full rotation } \\
\text { clockwise }\end{array}$ & $\begin{array}{c}\text { The automatic } \\
\text { faucet stop water } \\
\text { and soap from } \\
\text { running }\end{array}$ \\
\hline
\end{tabular}

\subsection{Validation by health practitioner}

In Validation Test, Health practitioner was asked to perform hand-wash using the developed automatic faucet. The result showed that it was conformed the hand-wash standard procedure which emphasizes usage of soap and scrubbing duration of 20 seconds. Figure 7 shows a Health Practitioner used the faucet for hand-washing.

\subsection{User-participation test}

The User-Participation Test consisted of two test, which were: (1) Observation on users' execution time of handwashing using conventional faucet with separated soap and the developed automatic faucet; (2) Usability Evaluation by users using USE Questionnaire. Total 30 respondents range from teenager to elderly people was gathered in correlation with the definition of users in "What is Wanted". 


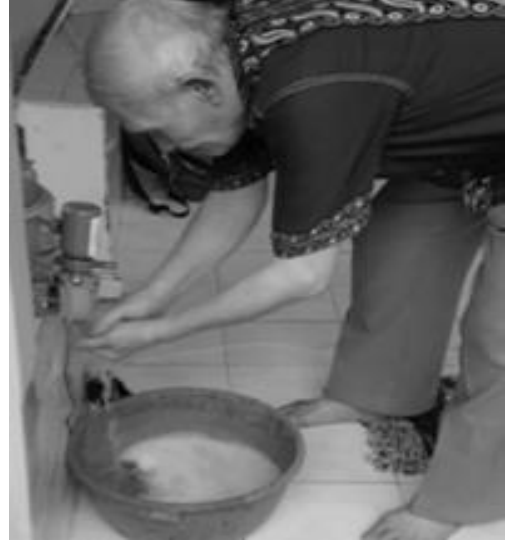

Fig 7. A healthcare practitioner used the automatic faucet for hand-washing

\subsubsection{Observation on user's execution time}

In the test, users where asked to wash their hands using a conventional faucet and then the automatic faucet. Since there are no guidance for exact duration of scrubbing in the conventional faucet, users scrubbed their hands varied in 4 to 44 seconds (average 15.4 seconds). This duration is less than the standard. Whilst, the automatic faucet guided the users to scrub their hands in exactly 20 seconds in accordance with the standard rules. The automatic faucet were also successfully forced the users to wash their hands with soap. They also directly scrubbed their hands with the soap intuitively.

\subsubsection{Usability evaluation using USE questioner}

USE questionnaire is generally used to evaluate user attitudes towards a variety of consumer products using three dimensions: Usefulness, Satisfaction, and Easiness (Ease of Use and Ease of Learn) [17]. It consists of 30 questions where users answer using Likert Scale of 1 to 7. This scale is often found in the questionnaire to facilitate the respondents to give answers in structured and limited options [18]. The scale used in this test is a scale of 1 (strongly disagree), 2 (disagree), 3 (rather disagree), 4 (doubtful), 5 (rather agree), 6 (agree), and 7 (strongly agree). A statistical parameter of mode is commonly used to analyse categorical data from Likert scale as used in the USE questionnaire.

The result shows a mode of grade 7 for Usefulness, grades 6 for Satisfaction, grades 6 for Easy to Use, and grade 6 for Easy to Learn. The mode of all usability criteria is grade 6 which mean users "agree" that the developed automatic faucet has maximized its usability.

\section{Conclusion}

In this paper, a fully automatic faucet for hand-washing has been developed using Interaction Design process to maximize its usability. The analysis on Verification, Validation and User-Participation test has shown that it functions well to force users to use soap in their handwashing routine and to scrub in 20 seconds as well as excel the usability in terms of Usefulness, Satisfaction and
Easiness. Further development should focused on optimizing the hardware, housing and electrical source. An addition of dryer should also be designed to meet the hand-washing standard that includes drying activity.

\section{References}

1. D. Pittlet, WHO Guidelines on Hand Hygiene in Helath Care: a Summary. World Health Organization Patient Safety: University of Geneva Hospitals (2009)

2. A. E. Aiello, R. B. Coulborn, V. Perez, and E. L. Larson, Effect oh Hand Hygiene on Infectious Disease Risk in the Community Setting: A-MetaAnalysis, in American Journal of Public Health, 98(8), 1372-1381 (2008)

3. M. Lovely, Disease of Dirty Hands. Medicine Adviser: your health (2013)

4. E. N. Perencevich, M. T. Wong, and A. D. Harris, National and Regional Assessmnt of the Antibacterial Soap Market: A Step Toward Determining in the Impact of Prevalent Antibacterial Soap, In American Journal of Infection Control, 29(5), 281-283, (2001)

5. A. E. Aiello and E. L. Larson, What is the Evidence for a Causal Link between Hygiene and Infections, In Lancet Infectious Diseases, 2(2), 103-110, (2002).

6. K. B. Kamm, D. R. Feikin, G. M. Bigogo, G. Aol, M. M. Shah, J. Yu, R. F. Breiman, and P. K. Ram, Associations between Presence of Handwashing Stations and Soap in the Home and Diarrhoea and Resiratory Illness, in Children Less than Five Years Old in Rural Western Kenya, In Tropical Medicine and International Health Journal, 19(4), 398-406, (2014)

7. Mayo Clinic Staff, Hand-washing: Do's and Don'ts. Healthy Lifestyle Adulth Health (2014)

8. M. Burton, E. Cobb, P. Donachie, G. Judah, V. Curtis and W. P. Schmidt, The Effect of Handwashing with Water or Soap on Bacterial Contamination of hands, In International Journal of Environmental Research and Public Health, 8(1), 97-104 (2011)

9. V. Boscart, K. McGilton, A. Levchenko, G. Hufton, P. Holliday, and G. Fernie, Acceptability of a wearable hand hygiene device with monitoring capabilities, Journal of Hospital Infection, 70, 216222 (2008)

10. J. M. Boyce and D. Pittet, Guideline for Hand Hygiene in Health-Care Settings: Recommendaions of the Healthcare Infection Control Practies Advisory Comitte and the HICPAC/SHEA/APIC/IDSA Hand Hygiene Task Force. Department of Health and Human Services: Morbidity and Mortality Weekly Report, USA (2002)

11. A. Dix, F. Janet, D. Gregory . B. A. Rusell, Human Computer Interaction, $3^{\text {rd }}$ ed., Haddigton: Scotprint (2004)

12. J. H. Goldberg and X. P. Kotval, Computer Interface Evaluation using Eye Movement: Methods and Constructs, in International Journal of Industrial Ergonomics, 24(6), 631-645, (1999) 
13. A. Dix, Beyond Interaction Pushing Boundaries with Incidental Interaction: Lancaster University, UK (2002)

14. A. Bianchi, D. M, WHO Guidelines on Hand Hygiene in Helath Care: a Summary. World Health Organization Patient Safety: University of Geneva Hospitals (2009)

15. E. Stanley and Sr. Flowers, Automatic Hand Washing and Drying Machine, U.S. Patent US5924148A (1999)

16. C. P. Borchgrevink, J. Cha and S. Kim, Hand Washing Practices in a Collage Town Environment, Journal of Environ Health, 75, 18-24 (2013)
17. A. M. Lund, Measuring Usability with the USE Questionnaire, STC Usability SIG Newsletter, 2-8, (2001)

18. R. A. Likert, A Technique for The Measurement of Attitudes, Archives of Psychology, 140, 1-55 (1993) 